\title{
Real-World Outcomes Among US Veterans Health Administration Patients Newly Diagnosed with Metastatic Renal Cell Carcinoma and Treated with First-Line Monotherapy
}

\author{
Abhijeet Bhanegaonkar · Shivani Pandya · Ying Zheng • \\ Ruth Kim • Stan Krulewicz • Vijay Kasturi · Hemant Phatak
}

Received: October 29, 2020 / Accepted: February 4, 2021 / Published online: April 17, 2021

(C) The Author(s) 2021

\begin{abstract}
Introduction: Substantial unmet needs exist among patients with metastatic renal cell carcinoma (mRCC). This retrospective study evaluated treatment patterns as well as clinical and economic outcomes associated with first-line monotherapy among patients with mRCC in the USA.
\end{abstract}

Methods: Newly diagnosed patients with mRCC initiating at least one first-line therapy (1L) from 1 October 2013 to 31 March 2018 (index date $=1 \mathrm{~L}$ start date) were identified from the US Veterans Health Administration database. Treatment patterns, clinical outcomes (time to next treatment [TNT] defined by earliest of switch to non-index therapy or re-initiation of index therapy after a more than 90-day gap, time to treatment discontinuation [TTD], overall survival $[\mathrm{OS}]$ ), and costs were evaluated

A. Bhanegaonkar $(\bowtie) \cdot$ Y. Zheng · V. Kasturi .

H. Phatak

EMD Serono Inc., Rockland, MA, USA; an affiliate of

Merck KGaA, Darmstadt, Germany

e-mail: abhijeet.bhanegaonkar@emdserono.com

S. Pandya

STATinMED Research, Plano, TX, USA

R. Kim

Pfizer, Inc., New York, NY, USA

S. Krulewicz

Pfizer, Inc., Collegeville, PA, USA among patients treated with tyrosine kinase inhibitors (TKI), mammalian target of rapamycin inhibitors (mTOR), immune checkpoint inhibitors (ICI), and other monotherapies. Standard descriptive statistics were presented. The Kaplan-Meier method was used to evaluate clinical outcomes.

Results: Of 759 patients (median age 68.0 years), $85.0 \%, 8.0 \%, 4.3 \%$, and $2.6 \%$ were treated with TKI, mTOR, ICI, or other therapy in $1 \mathrm{~L}$, respectively. Advancement rates (to secondline [2L] therapy) ranged from 10.0 (ICI) to 45.1 per 100 person years (TKI). The 12-month OS rates ranged from $47.4 \%$ (TKI) to $67.7 \%$ (mTOR). The median TNT ranged from 3.8 (mTOR) to 9.6 months (ICI), and median TTD ranged from 2.3 (mTOR) to 4.7 months (TKI). Total all-cause mean costs per patient per month ranged from $\$ 12,466$ (mTOR) to $\$ 19,812$ (ICI).

Conclusion: These results indicate high unmet medical needs among patients with mRCC treated with $1 \mathrm{~L}$ monotherapies. Novel combination therapies (e.g., ICI + ICI, ICI + TKI) may improve front-line outcomes for patients with poor prognoses.

Keywords: Health care resource utilization; Health economics and outcomes research; Immune checkpoint inhibitors; Metastatic renal cell carcinoma; Nephrology; Oncology; Tyrosine kinase inhibitors 
Key Summary Points

Why carry out this study?

Renal cell carcinoma (RCC) is the most common form of kidney cancer, with a poor prognostic outlook that makes it a public health concern.

The vast majority of RCC first-line (1L) monotherapy patients have unmet needs.

This study evaluated treatment patterns and patient outcomes among patients with metastatic RCC treated with the three most common monotherapy options.

\section{What was learned from the study?}

The study confirmed and described the unmet needs through observations of suboptimal treatment patterns as well as consistently high morbidity, mortality, and costs across treatments.

The results suggest value in continued investigation of novel combination therapies as an alternative to monotherapy in $1 \mathrm{~L}$.

\section{DIGITAL FEATURES}

This article is published with digital features, including a summary slide, to facilitate understanding of the article. To view digital features for this article go to https://doi.org/10.6084/ m9.figshare.13713025.

\section{INTRODUCTION}

Renal cell carcinoma (RCC) is the most common form of kidney cancer: it accounts for approximately $90 \%$ of all renal cancers in the USA and is the third most common urologic malignancy worldwide $[1,2]$. The disease is most prevalent among older adults, with a median age of 65 years at onset in the USA [3]. Overall worldwide incidence and mortality rates are increasing at a rate of approximately $2-3 \%$ per decade [4], with the highest estimated incidence in North America (age-standardized rates 12 per 100,000 ), and cumulative risks of $1.8 \%$ and $0.9 \%$ for men and women, respectively [5]. However, the incidence rates in the USA have stabilized since 2008, and US mortality rates have decreased at accelerating rates from 2001 through 2015; rates in Western nations at large show similar downward trends [6]. Nonetheless, in 2020, an estimated 73,750 individuals in the USA will be diagnosed with some form of renal cancer, which will cause 14,830 deaths [7]; thus, all renal cancer-and especially RCC-remains a considerable concern.

This concern stems in large part from the potential for progression to metastasis and the poor prognostic outlook for patients who do progress. As RCC is initially asymptomatic, $25-30 \%$ of patients have metastatic disease by the time of diagnosis [4]. Moreover, less than $5 \%$ have solitary metastasis, and overall, metastatic RCC (mRCC) is counted among the most treatment-resistant malignancies [4]. Median survival is approximately 1 year after diagnosis, with response rates of approximately 15-25\% for treated patients overall [4]. The 5-year survival rate for mRCC is only $11.1 \%$ in contrast to $90.8 \%$ for local disease and $63.3 \%$ for regional disease [8]. Although partial or total nephrectomy is a viable treatment option for localized RCC, approximately $20-30 \%$ of patients with limited disease progression at the time of surgery still develop metastasis [9].

Historically, therapeutic options for patients with mRCC have been limited, with first-generation cytokine immunotherapies such as interferon- $\alpha$ and interleukin- 2 representing the standard of care through 2005 [10]. However, therapies have evolved substantially within the last two decades, as the low response rates and side effects associated with immunotherapy have necessitated the development of more effective targeted therapies [10-12]. These have included vascular endothelial growth factor (VEGF) inhibitors and mammalian target of rapamycin inhibitors (mTOR), both of which 
have been associated with higher survival rates as compared with first-generation cytokine immunotherapy [9].

At the time of this study's analysis, standard of care monotherapies included mTOR inhibitors as well as multitargeted tyrosine kinase inhibitors (TKIs), a subcategory of VEGF inhibitors. Both monotherapies have shown improved progression-free survival (PFS), efficacy, and increased median survival time as compared with cytokine immunotherapy $[10,13,14]$. While these agents have been associated with improved palliative outcomes, the rarity of complete remission has driven continued pursuit of alternatives, including a resurgence of clinical interest in immunotherapy [10]. Specifically, immune checkpoint inhibitors (ICI) have presented promising firstline $(1 \mathrm{~L})$ treatment options in mRCC, first as monotherapy and then in combination with other agents with different mechanisms of action (including ICI + ICI and ICI + TKI combinations) $[15,16]$.

ICI, which constitute the majority of immunotherapies utilized today, are more tolerable than their cytokine therapy predecessors and have demonstrated superior PFS and overall survival (OS) in mRCC [10]. The National Comprehensive Cancer Network Clinical Practice Guidelines in Oncology now recommend these targeted treatments paired together and with TKIs as $1 \mathrm{~L}$ combination therapies $[17,18]$.

However, despite the availability of targeted combination treatment options, many patients with RCC still receive monotherapy in the $1 \mathrm{~L}$; of these patients, a clear majority have substantial unmet needs. Objective response rates in $1 \mathrm{~L}$ have ranged from approximately $12 \%$ to $50 \%$ overall, including patients with differing risk levels and histologies [19]. Patients with inferior prognoses based on metastatic site are particularly vulnerable $[10,11]$; e.g., only a small proportion of patients have achieved complete responses and durable response rates greater than 12 months [10].

The most recent review of the overall economic impact of RCC in the USA estimated costs of approximately $\$ 5.7$ billion, with yearly costs of $\$ 51,930$ per patient (adjusted to 2018 US dollars) [20]. Moreover, these numbers are expected to increase because of the aging population and increasing prevalence of risk factors associated with RCC such as obesity and hypertension $[2,21]$.

Together with high mortality, the profound economic burden of the disease and increasing heterogeneity of treatment options underscore the importance of optimizing $1 \mathrm{~L}$ treatment, and require close scrutiny of the reliance on monotherapy in the 1L. This includes continuing investigation of real-world treatment patterns and correlated outcomes to complement randomized controlled trials of novel agents. Therefore, the current study was conducted with two main objectives: to describe the treatment patterns and clinical outcomes of patients with mRCC treated with $1 \mathrm{~L}$ systemic monotherapy, and to evaluate health care resource utilization (HCRU) and costs among the same population. Toward this end, the study leveraged US Veterans Health Administration (VHA) claims data because the VHA population is at higher risk for mRCC, as it is primarily comprised of older men with high rates of obesity, smoking, and hypertension [22-25].

\section{METHODS}

\section{Data Source}

This retrospective observational study utilized data from the VHA from April 1, 2013 through March 31, 2018 to assess the treatment patterns, clinical outcomes, and economic outcomes (HCRU and costs) of patients with mRCC. The VHA is the largest integrated health care system in the USA, providing a broad spectrum of health care services to over 9 million enrollees across the country. Care is provided at 1250 health care facilities, including 172 VA medical centers and 1069 outpatient sites of care [26]. The data for this study were extracted from the VHA Medical Inpatient (including inpatient stay, specified physician, procedures, and surgeries), Outpatient (including single day-visit data from clinics, laboratories, and treatment programs), and VHA Decision Support System (DSS) datasets. The VHA DSS is a longitudinal, 
relational database that combines the clinical and cost data needed to integrate expenses and utilization of various services and allocate the costs to appropriate services provided to veterans [27]. Only patients with complete data were included in the raw dataset, thus no exclusions or adjustments for incomplete data were necessary.

\section{Selection Criteria}

Patients aged 18 years or more were included if they were diagnosed with RCC (The International Classification of Diseases, Ninth Revision, Clinical Modification [ICD-9-CM]: 189.0, 189.1; ICD-10-CM: C64, C65) anytime during the study period from April 1, 2013 through March 31,2018 and if they had at least one subsequent medical claim for metastasis (ICD-9-CM: 196, 197, 198, 199; ICD-10-CM: C77, C78, C79, C45.9) from October 1, 2013 through March 31, 2018. The first metastatic diagnosis date was defined as the mRCC diagnosis date. Included patients must have received at least one mRCC single-agent systemic or oral therapy on or after the initial mRCC diagnosis date, with the first therapy claim date identified as the index date and also marking the start of 1L monotherapy. Patients were further required to have continuous VHA medical and pharmacy enrollment from at least 6 months prior to the mRCC diagnosis date through at least 6 months postindex date (patients who died before 6 months after index were included in the analysis). Patients were followed until the earliest of plan disenrollment, death, or study end.

Patients were excluded if they had evidence of any mRCC systemic therapy during the 6-month pre-index (baseline) period, a diagnosis for cancer other than RCC in the primary or secondary position within 6 months before the mRCC diagnosis date, pregnancy during the study period, TKI-TKI or TKI-mTOR combination therapy, or death within 14 days of the index date. The 6-month period prior to the index date was defined as the baseline period.

The patients were assigned to four mutually exclusive monotherapy cohorts based on $1 \mathrm{~L}$ systemic therapy regimens: TKI, mTOR, ICI, or other.

\section{Compliance with Ethics Guidelines}

This retrospective database analysis did not involve the collection, use, or transmittal of individual identifiable data. As such, institutional review board approval to conduct this study was not required, as it is considered exempt according to 45CFR46.101(b)(4): Existing Data \& Specimens-No Identifiers. Both the dataset itself and the security of the offices where the data are housed meet the requirements of the Health Insurance Portability and Accountability Act of 1996.

\section{Study Measures}

\section{Patient Characteristics}

Demographic characteristics such as age, sex, and racial distributions were examined on the index date. The comorbidity burden during the baseline period was examined using two indices: the Quan-Charlson comorbidity index (CCI) score, which assesses 17 comorbidities [28], and the National Cancer Institute (NCI) comorbidity index score, a cancer-specific version of the CCI that excludes solid tumors, leukemia, and lymphoma [29].

In addition, all-cause HCRU and RCC-related costs per patient per month (PPPM) during the baseline period were calculated for inpatient, outpatient, and pharmacy services. Moreover, total medical (inpatient + outpatient) and total (medical + pharmacy) costs were calculated.

\section{Treatment Patterns}

\section{Line of Therapy Definitions}

The lines of therapy were determined using a claims data algorithm based on the start and end dates of a treatment regimen. The $1 \mathrm{~L}$ systemic therapy was considered to have started on the index date, and all systemic therapies within 14 days of the index date were designated as part of the $1 \mathrm{~L}$ systemic therapy. The start of second-line (2L) systemic therapy was defined by the earliest of addition of/switch to a 
non-index systemic therapy or re-initiation of the index therapy after a more than 90-day gap from the end of prior therapy. The duration of $1 \mathrm{~L}$ therapy was defined as the period from the index date until the earliest of the end date of the last index prescription's days of supply, a day prior to the start of $2 \mathrm{~L}$ therapy, or follow-up end. The patients were categorized as having continued, switched, interrupted, or permanently discontinued therapy as follows:

Switched Patients who switched to or added on a non-index medication with or without a more than 90-day gap in therapy.

Interrupted Patients whose 1L treatment was halted and then reinitiated after the end of the last index prescription's days of supply plus a more than 90-day gap with no non-index mRCC systemic therapies before or upon re-initiation.

Permanently Patients who ceased their index discontinued systemic therapy with no switch to a non-index mRCC systemic therapy and no index reinitiation from the last index prescription through follow-up.

Continued Patients who did not switch, interrupt, or discontinue (as defined above) their index systemic therapy until the earliest of disenrollment, study end, or death.

\section{Clinical Outcomes}

The clinical outcomes evaluated in this study included time to treatment discontinuation (TTD), time to next treatment (TNT), and OS. TTD was measured as the period (in months) from $1 \mathrm{~L}$ systemic therapy initiation until the earliest of 1L discontinuation or death. TNT was measured as period (in months) from 1L systemic therapy initiation until the earliest of $2 \mathrm{~L}$ therapy initiation or death. OS was defined as the time from $1 \mathrm{~L}$ systemic therapy initiation until death or follow-up end. The 12-month OS rate was also reported.

\section{Economic Outcomes}

All-cause and mRCC-related HCRU and costs PPPM were calculated for inpatient, outpatient, and pharmacy services and reported for the duration of 1L therapy. Direct health care costs were evaluated from a payer perspective and covered cost for all care received in the inpatient, outpatient, and pharmacy settings. Inpatient visits (including length of stay [LOS]) and expenditures included the care received in the acute care and extended care settings (e.g., nursing homes). Outpatient visits and expenditures included the care received in any outpatient setting, including outpatient office visits, emergency department visits, laboratory services, and all other services. Pharmacy visits and expenditures included prescriptions from inpatient and outpatient pharmacy. All inpatient and outpatient claims on a day with an mRCC diagnosis were defined to be mRCC related. All pharmacy claims for mRCC systemic therapy were also defined to be mRCC related. In addition, total medical (inpatient + outpatient) and total (medical + pharmacy) costs were calculated.

\section{Statistical Analysis}

The current study employed an "as-treated" analysis model. All study variables, including patient demographics and clinical characteristics as well as clinical and economic outcomes, were described with standard summary statistics among patients treated with different $1 \mathrm{~L}$ monotherapies. Categorical variables were summarized in the form of numbers and percentages. Continuous variables were summarized in the form of means and standard deviations (SDs). The rates of switching, continuing, interrupting, and permanently discontinuing were reported in 100 person years (PYs). These were calculated as the number of patients with events of interest divided by PY at risk for developing the event (divided by 100).

Survival analysis using Kaplan-Meier (KM) curves was conducted to assess the median TNT, TTD, and OS (in months) within treatment cohorts only without statistical comparisons. The event-free probabilities for TNT and TTD 
from the KM curves were also reported at 6,12 , and 18 months from the index date.

Given the variable duration of 1L therapy for each patient, HCRU and costs were reported on a PPPM basis by first dividing each patient's total visits and costs accrued during the duration of $1 \mathrm{~L}$ therapy by the duration of $1 \mathrm{~L}$ therapy (in months) and then calculating the group average for all individual patient averages. The costs were adjusted to 2018 US dollars using the medical care component of the Consumer Price Index from the US Department of Labor.

\section{RESULTS}

\section{Study Population}

A total of 759 patients with mRCC met the selection criteria. Of these, 645 (85.0\%) were treated with TKIs, 61 (8.0\%) were treated with mTORs, 33 (4.3\%) were treated with ICIs, and the remaining $20 \quad(2.6 \%)$ received other monotherapies (Fig. 1). As a result of the low sample size of the other therapy cohort, characteristics and outcome results for this group are not reported here. Patients in the TKI, mTOR, and ICI cohorts received monotherapies of agents in their respective drug classes.

\section{Baseline Characteristics of Patients with mRCC Treated with 1L Systemic Monotherapy}

Across cohorts, patients were nearly all male (approximately $98 \%$ ), and predominantly older and white; average ages ranged from 66.2 to 68.2 , with large majorities in all cohorts aged at least 65 years, and white patients accounting for $57.6 \%$ to $81.2 \%$. All cohorts had a substantial comorbidity burden as indicated by the Quan-CCI and NCI scores, and rates of selected individual comorbidities. QuanCCI score averages were severe $(>6)$ across cohorts and ranged from 8.8 to 9.6, and NCI averages ranged from 1.9 to 2.2 (Table 1 ).

Baseline HCRU was considerable, with the average number of baseline all-cause PPPM inpatient visits ranging from 0.2 to 0.3 . The average inpatient LOS PPPM ranged from 0.7 to
1.1 days, and mean outpatient visits PPPM ranged from 2.9 to 4.2. Mean pharmacy visits PPPM ranged from 2.7 to 4.2 . In line with utilization, costs were also substantial, with total mean costs ranging from $\$ 4529$ PPPM (TKI) to $\$ 12,681$ PPPM (ICI). Costs were primarily driven by inpatient and outpatient visits, with the exception of relatively high pharmacy costs among the ICI cohort (\$4011) (Table 2).

\section{Treatment Patterns}

Rates of treatment continuation ranged from 20.5 to 53.0 per 100 PY for TKI and ICI, respectively; treatment interruption from 2.6 to 10.1 per 100 PY for mTOR and ICI, respectively; and permanent discontinuation from 25.5 to 62.0 for TKI and ICI, respectively. Switching to $2 \mathrm{~L}$ therapies occurred in all cohorts but varied considerably, ranging from 10.0 to 45.1 per 100 PY for ICI and TKI, respectively (Fig. 2). The median duration of $1 \mathrm{~L}$ therapy was $3.6,6.5$, and 7.0 months among patients treated with mTOR, ICI, and TKI therapy, respectively.

\section{Clinical Outcomes}

Key clinical outcomes estimated from KM curves varied across cohorts, with more consistency in generally short mean TTD and TNT ranges (and therefore also the implied range for approximate estimation of actual progressionfree survival). The 12-month OS rates, as estimated from the KM curves, ranged from $47.4 \%$ (mTOR) to $67.7 \%$ (TKI). Median OS ranged from 10.8 (mTOR) to 25.1 months (TKI). Median TNT ranged from 3.8 (mTOR) to 9.6 months (ICI). Median TTD ranged from 2.3 (mTOR) to 4.7 months (TKI) (Fig. 3).

Event-free probabilities for TTD ranged as follows: 6 months-14.8\% (mTOR) to $41.7 \%$ (TKI); 12 months-24.2\% (mTOR) to $24.2 \%$ (TKI); and 18 months-4.9\% (mTOR) to $12.7 \%$ (TKI). The 6-, 12-, and 18-month event-free probabilities for TNT followed similar trends, and ranged as follows: 6 months $-34.4 \%$ (mTOR) to $60.0 \%$ (TKI); 12 months-26.2\% (mTOR) to $43.8 \%$ (ICI); and 18 months-21.3\% (mTOR) to $35.0 \%$ (TKI). 
Patients diagnosed with mRCC during the identification period from October 1, 2013 through March 31, 2017; the first metastatic diagnosis date was defined as the mRCC diagnosis date.

$N=6,071$

Patients with evidence of any mRCC systemic therapy on or after the initial mRCC diagnosis date during the indentification period. The first therapy claim date was designated as the index date and was considered the initiation of $1 \mathrm{~L}$ therapy.

$\mathrm{N}=2,197$

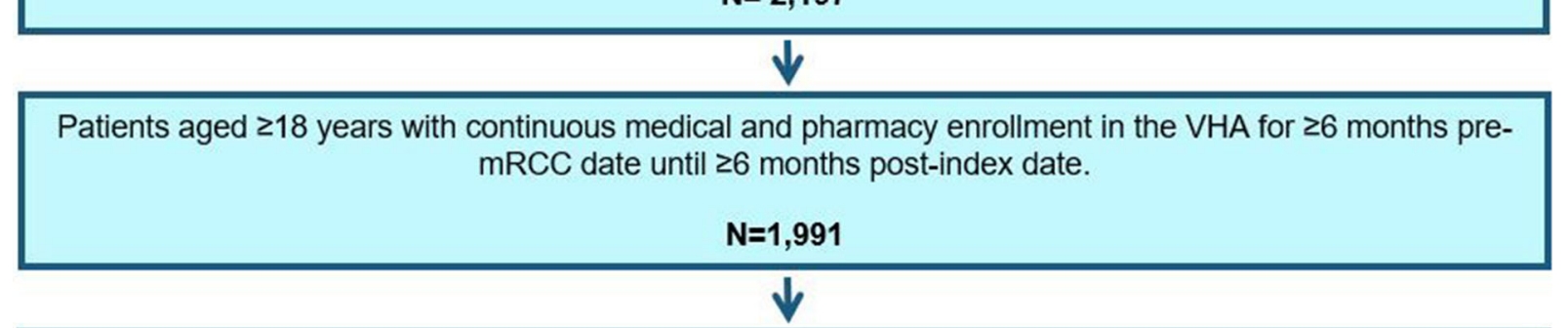

Patients without: any $\mathrm{mRCC}$ systemic therapy during the 6 months prior to the index date; evidence of pregnancy duiring the study period; diagnosis for cancer other than RCC $\leq 6$ months pre-mRCC diagnosis date; evidence of death within 14 days post-index date; or TKI -TKI or TKI - mTOR combination therapy.

$\mathrm{N}=759$

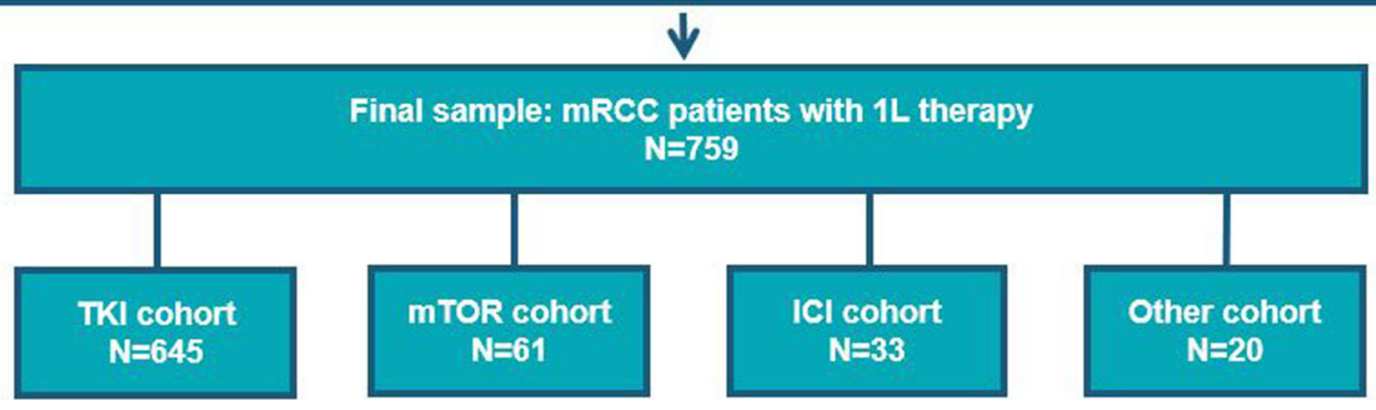

Fig. 1 Patient selection and attrition. 1L, first-line; ICI, immune checkpoint inhibitor; mRCC, metastatic renal cell carcinoma; mTOR, mammalian target of rapamycin; TKI, tyrosine kinase inhibitor; VHA, Veterans Health Administration. Note: TKIs included pazopanib,

sunitinib, axitinib, cabozantinib, sorafenib, lenvatinib, and erlotinib; mTORs included everolimus and temsirolimus; ICIs included nivolumab, ipilimumab, atezolizumab, and pembrolizumab; and the other cohort included interleukin, interferon, and bevacizumab

\section{HCRU and Costs Within the Duration of $1 \mathrm{~L}$ Treatment}

Overall HCRU and costs were considerable during the observed treatment periods, both driven primarily by mRCC-related events. Specifically, the average number of all-cause

inpatient visits PPPM ranged from 0.1 (ICI) to 0.4 (mTOR), and inpatient LOS ranged from 0.5 days (ICI) to 1.5 days (mTOR). The mean number of all-cause outpatient visits PPPM was high in all cohorts as well (TKI [3.6], ICI [4.6], and mTOR [4.9]). Similar results were noted for the number of pharmacy visits (Fig. 4). 


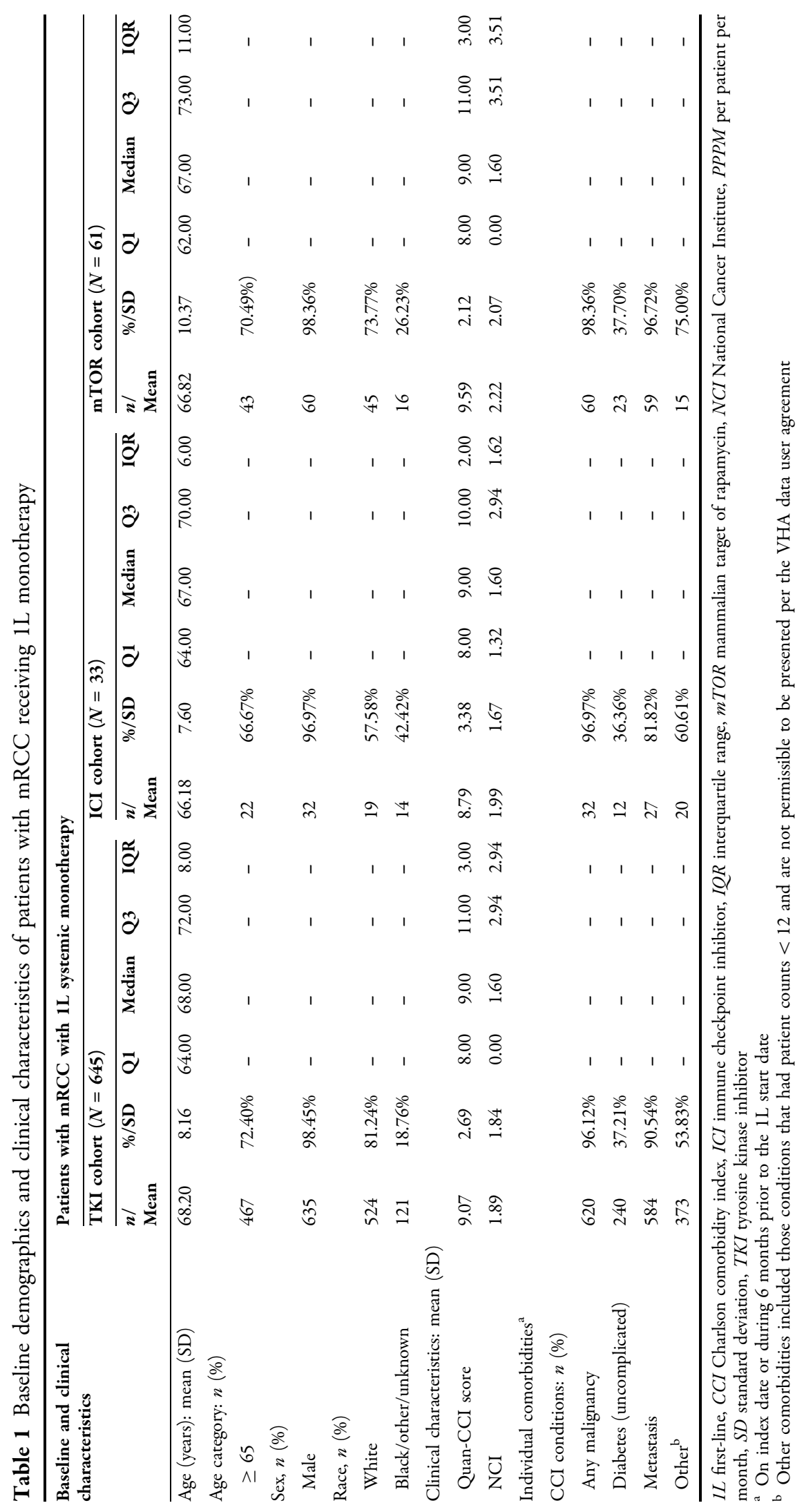




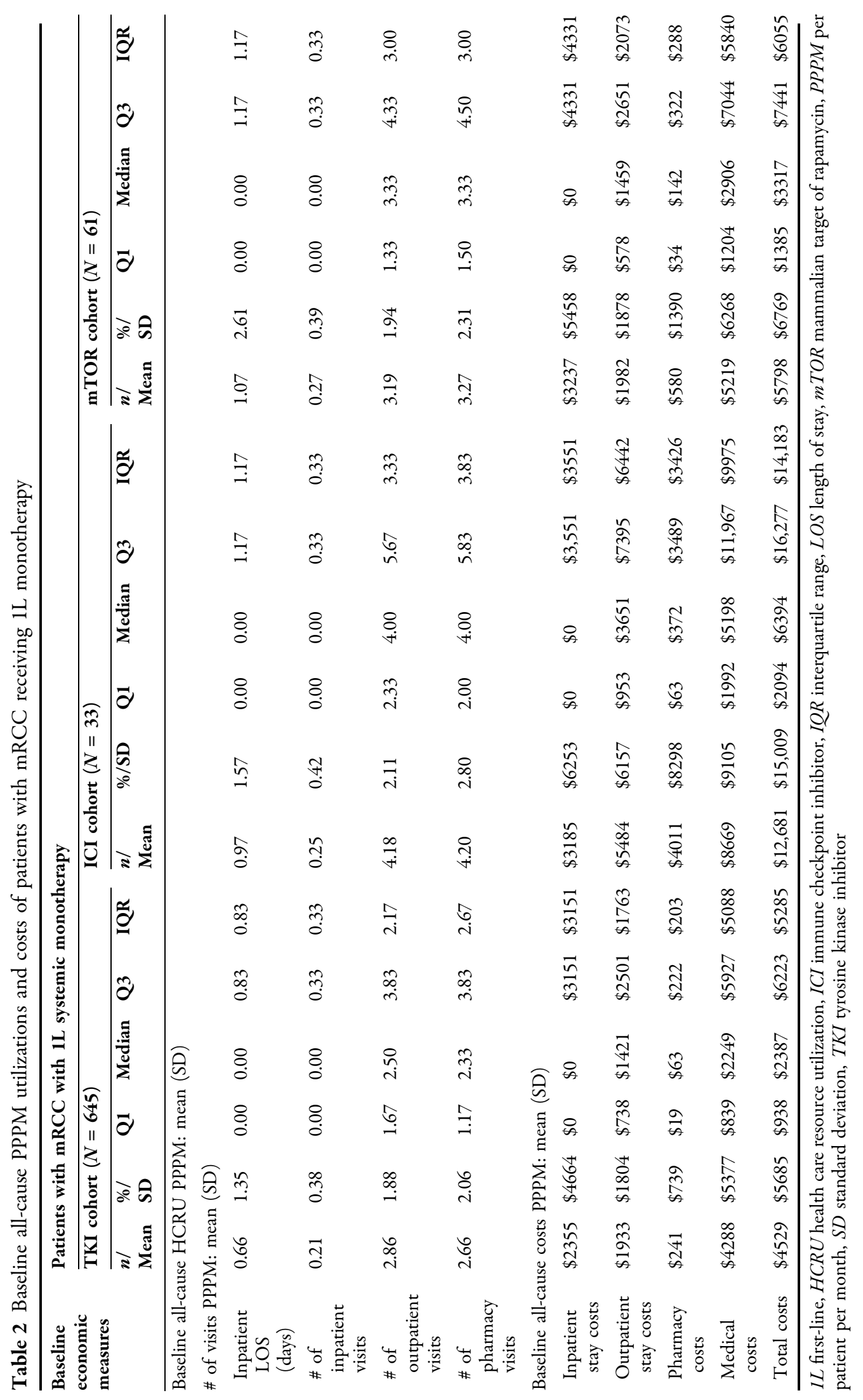



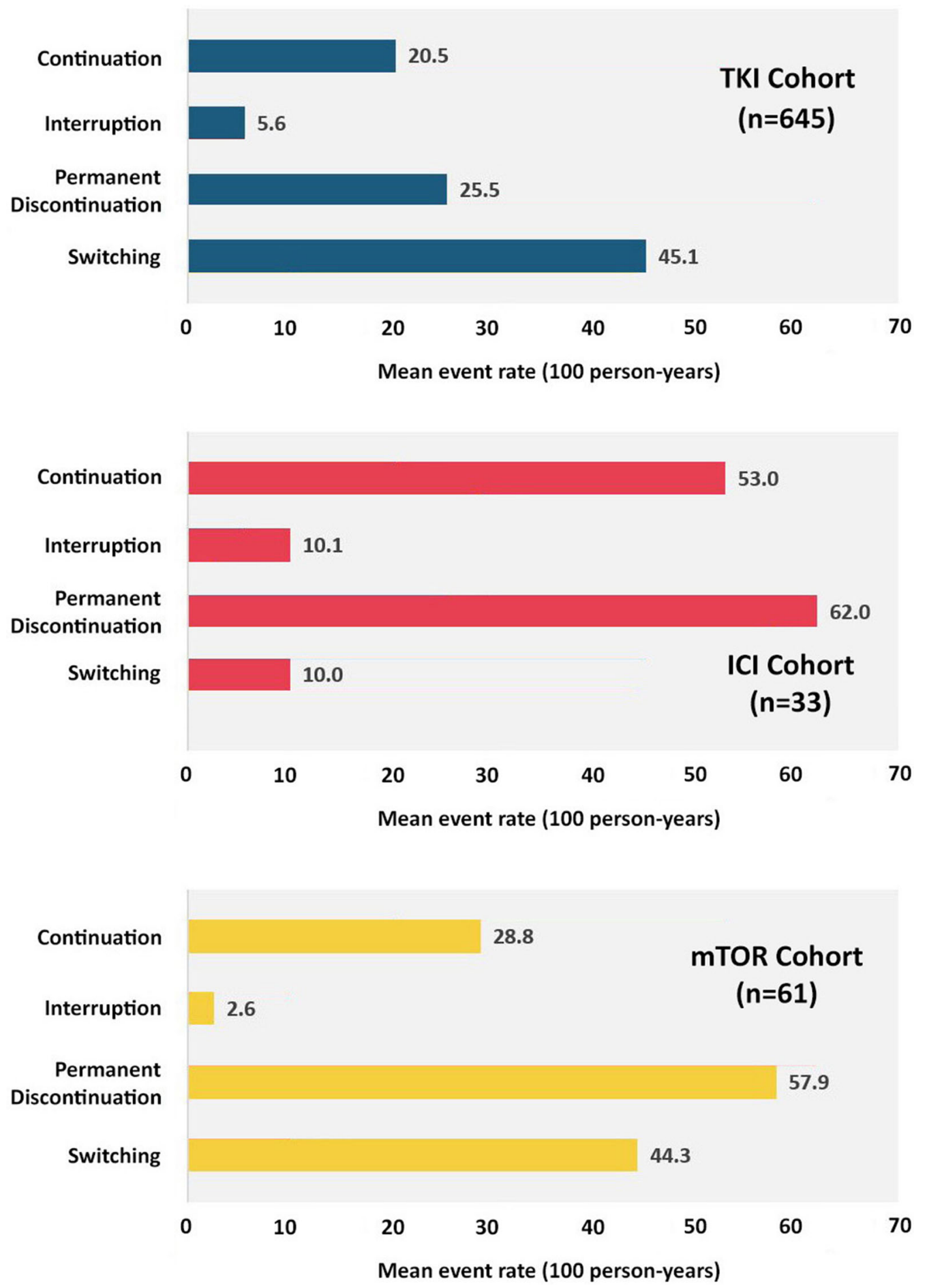

Fig. 2 Treatment patterns of patients with mRCC receiving IL monotherapy. 1L, first line; ICI, immune checkpoint inhibitor; mRCC, metastatic renal cell carcinoma; mTOR, mammalian target of rapamycin; TKI, tyrosine kinase inhibitor. Note: Results are descriptive only; cohorts were not adjusted for baseline patient characteristics 
These trends were largely attributable to mRCC-related HCRU. Across all cohorts, there were $0.1 \mathrm{mRCC}$-related inpatient visits, with mean LOS ranging from 0.4 (ICI) to 0.6 days (TKI/mTOR). Similar trends were noted for outpatient visits and pharmacy visits, with large proportions of total utilizations attributable to mRCC (Fig. 4).

In line with utilization, high costs were observed in all cohorts. Mean all-cause health care costs PPPM varied widely across cohorts, with total costs ranging from $\$ 12,466$ (mTOR) to $\$ 19,812$ (ICI). This broke down to a range of $\$ 6956$ (TKI) to $\$ 11,838$ (ICI) for total medical costs (inpatient plus outpatient; Fig. 5), and $\$ 5308$ (mTOR) to $\$ 7974$ (ICI) for pharmacy costs (Fig. 5).

Overall, most of the observed cost expenditures were attributable to mRCC-related costs, which varied between cohorts. Mean total
mRCC-related costs ranged from $\$ 10,504$ (mTOR) to $\$ 14,685$ (ICI); this was comprised of a range of $\$ 4788$ (TKI) to $\$ 6928$ (ICI) for total mRCC-related medical costs (inpatient plus outpatient) and \$4843 (mTOR) to \$7757 (ICI) for pharmacy costs (see Fig. 5).

\section{DISCUSSION}

To date, there have been no real-world studies examining the clinical and economic burden of treating mRCC among (primarily) older male US veterans, who are at increased risk of renal cancer due to the high prevalence of risk factors [23-25, 30]. Geynisman et al. [21] documented the clinical and economic burden experienced by US patients with mRCC being treated with 1L systemic or oral therapy. However, Geynisman et al.'s study was designed to measure treatment patterns more so than disease
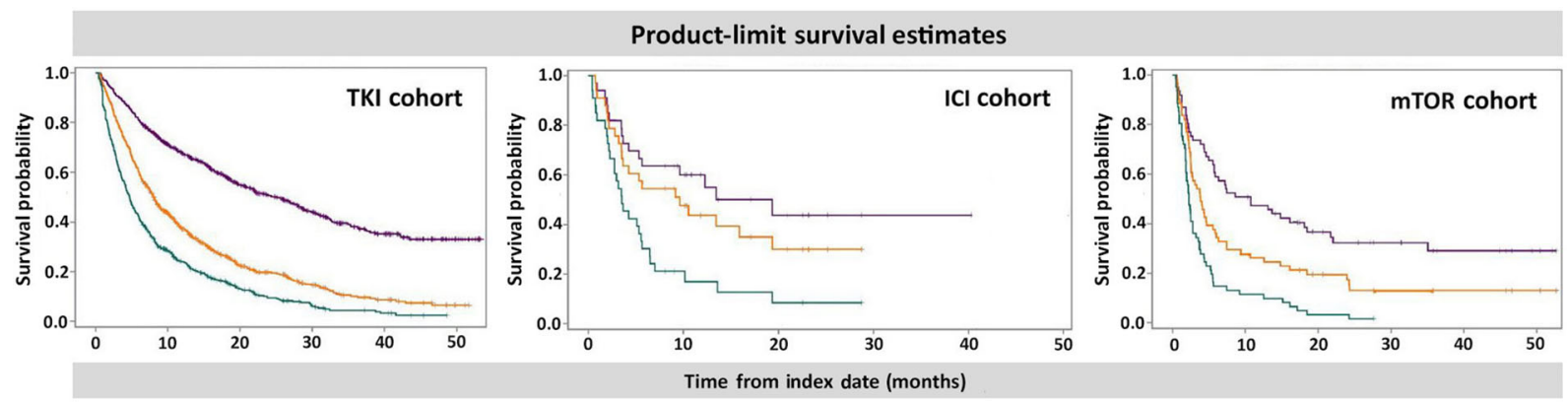

Clincial outcomes

TTD

TNT

os

Mean time-to-outcome estimates from KM curves (months)

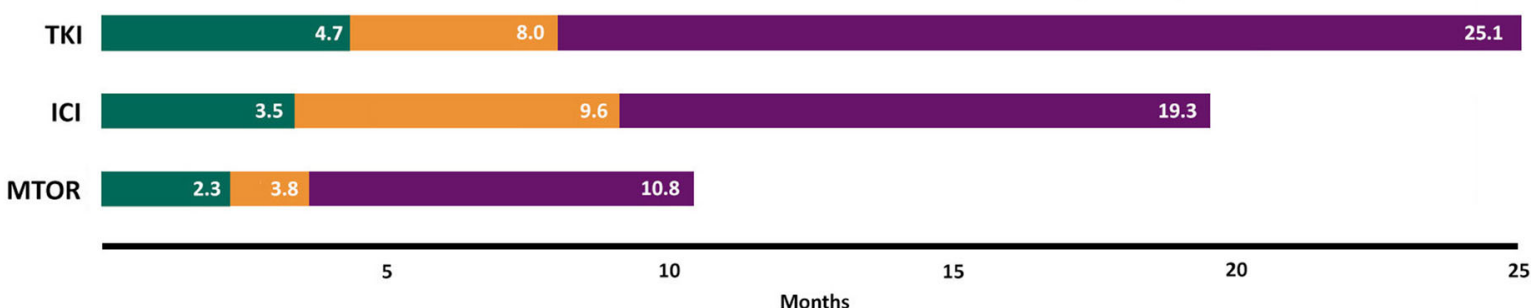

Months

Fig. 3 Kaplan-Meier curves and estimates for clinical outcome: 1L monotherapies. 1L, first line; ICI, immune checkpoint inhibitor; KM, Kaplan-Meier; mRCC, metastatic renal cell carcinoma; mTOR, mammalian target of rapamycin; OS, overall survival; PFS, progression-free survival; TKI, tyrosine kinase inhibitor; TNT, time to next treatment; TTD, time to treatment discontinuation. Note: Results are descriptive only; cohorts were not adjusted for baseline patient characteristics 

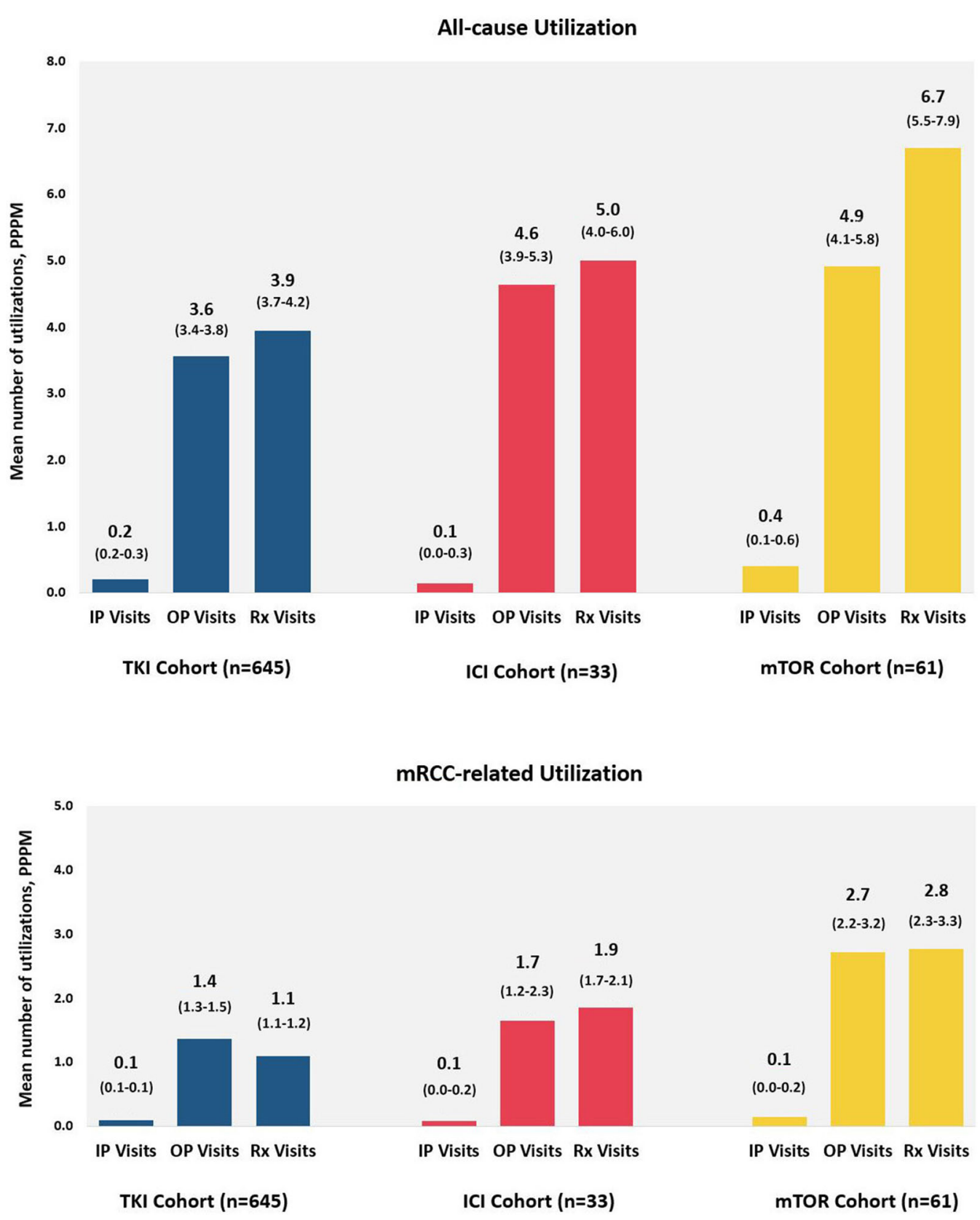

Fig. 4 Mean HCRU visits PPPM of patients with mRCC receiving various $1 \mathrm{~L}$ monotherapies. $1 \mathrm{~L}$, first line; HCRU, health care resource utilization; ICI, immune checkpoint inhibitor; IP, inpatient; mRCC, metastatic renal cell carcinoma; mTOR, mammalian target of rapamycin; OP,

progression, the population was limited to those aged less than 65 years, and newly recommended treatment options were not included, which makes the results difficult to generalize or compare with recent outpatient; PPPM, per patient per month; Rx, pharmacy; TKI, tyrosine kinase inhibitor. Note: Results are descriptive only; cohorts were not adjusted for baseline patient characteristics; 95\% confidence intervals (lower-upper limit) appear below mean results

developments in the literature [31-34]. To expand the limited evidence on patterns, progression, and burden, this study of a nationally representative population of patients at particular risk for mRCC provides insight into the 

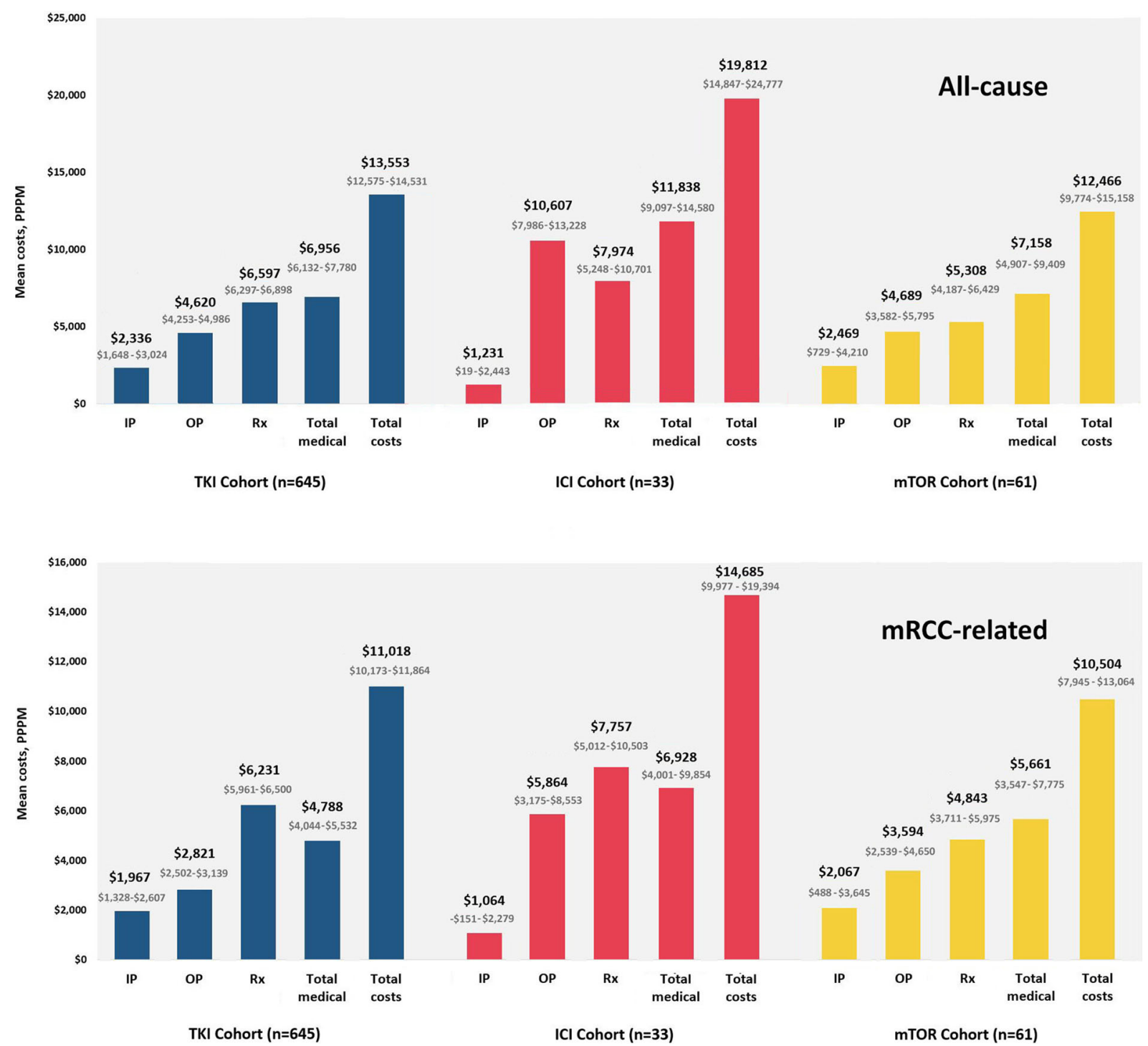

Fig. 5 Mean health care costs PPPM among patients with $m R C C$ receiving various $1 \mathrm{~L}$ monotherapies. $1 \mathrm{~L}$, first line; ICI, immune checkpoint inhibitor; IP, inpatient; mRCC, metastatic renal cell carcinoma; mTOR, mammalian target of rapamycin; OP, outpatient; PPPM, per patient per

effectiveness of current treatment patterns, and gaps in patient outcomes, in real-world clinical practice. As expected, patients were characterized by substantial morbidity at baseline; overall, the disease was responsible for considerable clinical and economic burden observed during treatment.

Our study revealed some underlying concerns regarding treatment patterns, specifically month; Rx, pharmacy; TKI, tyrosine kinase inhibitor. Note: Results are descriptive only; cohorts were not adjusted for baseline patient characteristics; 95\% confidence intervals (lower-upper limit) appear below mean results

1L monotherapy persistence. Results showed substantial rates of permanent discontinuation across all three reported cohorts and considerable switching among the TKI and mTOR cohorts. In addition, event rates of switching to $2 \mathrm{~L}$ therapies were high in this study population. The median duration of $1 \mathrm{~L}$ therapy was low, ranging from 3.6 months for those treated with mTORs to 7 months for those treated with TKIs. 
The economic burden associated with mRCC was also substantial in this study, but there is little recent real-world evidence on $1 \mathrm{~L}$ monotherapies in US patient populations with which to draw direct comparisons. Total costs in the present sample ranged from $\$ 12,466$ (mTOR cohort) to $\$ 19,812$ PPPM (ICI cohort), or approximately $\$ 150,000$ through $\$ 238,000$ yearly (2018 US dollars). As a historical reference, a 2011 study of SEER data (1991-2007) indicated that annual costs of treating older US patients with RCC with targeted therapies were considerably lower, ranging from approximately $\$ 75,000$ to $\$ 78,000$ (adjusted to 2018 dollars) [35]. However, the current study did not match cohorts to control for demographic and clinical confounders, and the studies differed substantively in their treatment pattern endpoints and interventions, as the previous data preceded the introduction of targeted therapies. More recent economic analyses have been marked by broad heterogeneity in cost definitions, populations, interventions, and parameters. Nonetheless, the present study results do generally align with a 2019 systematic literature review of the US economic burden of RCC, which observed rising costs overall and total $1 \mathrm{~L}$ annual costs of TKI monotherapies (in 2006-2014 datasets) ranging from approximately $\$ 73,000$ to $\$ 149,000$ (adjusted to 2018 US dollars), with a top range slightly lower than TKI results in the present study (approximately $\$ 163,000$ yearly) [36]. Some of this discrepancy could be due to variation in population and study design, but the present results warrant more sensitive investigation of whether the costs of treating the disease are continuing to increase, and if so, why.

Clinical outcomes were the main focus of the study and the main concern in view of the results. While it varied among treatment cohorts, the range of median OS in this sample-as estimated with KM curves-was generally in line with those observed in phase II and III clinical trials of, or including, 1L monotherapies, with median OS time ranging from approximately 21 to 29 months [31, 37, 38]. Among this sample, we noted median OS of roughly 19-25 months among the ICI and TKI cohorts, although it was somewhat lower among the mTOR cohort (ca. 10 months). Although PFS is a variable commonly studied in clinical trials, it was not directly capturable in the present study dataset. Nonetheless, this study was able to determine a general proxy for PFS by calculating TTD and TNT: real-world PFS would necessarily fall somewhere between $1 \mathrm{~L}$ discontinuation and 2L initiation (or end with death). Although there is presently little realworld evidence on $1 \mathrm{~L}$ monotherapies at the class level with which to compare, the present study found the range of times to progression considerably shorter as compared with clinical trials. For example, a 2011 review of six phase III trials found that median PFS ranged from approximately 4 to 11 months among mTORs and TKIs [39], whereas among these cohorts in the present study, the range of shortest TTD to longest TNT was 2.3-8.0 months; this suggests both a gap in patient outcomes between the two research spaces, and substantial unmet needs among this patient population.

Combination therapies present a viable alternative in clinical practice. In their 2018 manuscript of the phase III Checkmate 214 trial, Motzer et al. observed superiority of ICIICI combination therapy (nivolumab plus ipilimumab) with significantly higher objective response rates and $O S$ as compared with monotherapy (sunitinib) in the intermediate and poor risk groups over a median of 42 months of follow-up [31, 40].

In their 2020 interim analysis of the JAVELIN Renal 101 trial, Choueiri et al. compared an ICITKI combination (avelumab plus axitinib) with sunitinib monotherapy and found median PFS was significantly longer in the combination group (13.8 vs 7.0 months for patients with programmed death ligand-positive tumors, with median follow-up times of 19.3 and 19.2 months for the combination therapy and the monotherapy, respectively) [32]. This PFS range is considerably longer than the estimation from our results. Choueiri et al. also noted a superior objective response for the combination therapy as compared with the monotherapy in the trial as well as OS rates (over the aforementioned median follow-up times) superior to both trial comparators and the ranges in the present study. 
Rini et al. [34] noted similar trends in their 2019 phase III KEYNOTE-426 trial, with the target combination (pembrolizumab plus axitinib) significantly superior to sunitinib for median PFS (15.1 months vs 11.1 months, respectively) over a median follow-up time of 12.8 months. In the present study, the approximate monotherapy median PFS range (as estimated from the shortest median TTD to the longest median TNT) was considerably shorter at 2.3 months for mTOR through 9.6 months for ICI cohorts. Moreover, Choueiri et al.'s [33] analysis of event-free probabilities for TNT at 6 through 18 months showed superiority of a representative combination therapy range consistently throughout time (ca. 68\% [6 months; avelumab + axitinib] through ca. $48 \%$ [18 months]; visually presented) as compared with this study's monotherapy ranges (34.4-60.0\% [mTOR/TKI; 6 months] through 21.3-35.0\% [mTOR/ICI; 18 months]). These results suggest that novel combination therapies have considerable potential to improve outcomes among patients with poor prognoses treated with monotherapies.

There are several strengths of this study. The use of a representative, integrated dataset to analyze real-world patient outcomes by drug class provides results of interest in clinical practice. Further, the application of proxies to estimate PFS provides precedent for more sensitive analyses on a wider range of treatment options, including combination therapies. However, there are limitations to this study as well. Clinical conditions were identified using ICD-9-CM codes, with potential for miscoding. Algorithms were used to define lines of therapy based on administrative claims, which may not reflect the definition of lines of therapy used in clinical practice. Although the VA has relatively high proportions of this patient population and provides the largest integrated US datasets, results for VHA-enrolled patients with mRCC may have limited generalizability to the US patient population with differing insurance status and to other national patient populations. Clinical information regarding the rationale for treatment discontinuation and switching is not available in claims data. Moreover, information regarding differing histology was not in the current dataset. Further, as a result of sample sizes that varied widely and were small in two treatment cohorts, the study authors were unable to control for confounders and match cohorts for statistical comparison; thus, no tests for statistically significant differences were done, and the results for clinical and economic outcomes should be interpreted accordingly. Moreover, immunooncology (IO) plus TKI was not available as a 1L treatment option during the study period, as the analysis commenced before the recent approvals of TKI/IO combinations in the $1 \mathrm{~L}$ treatment of advanced RCC. Therefore, the results, while of clinical value, warrant future research with larger sample sizes that would support statistical comparison between treatment cohorts that include new combination therapies, with stratification for specific treatment options and prognostic risk factors such as histology.

\section{CONCLUSION}

Significant unmet needs were identified among this US patient population across all monotherapy classes with respect to OS, TNT, and TTD. Additionally, increased HCRU and costs were also noted among those treated with monotherapy. These outcomes indicate that current treatment strategies can be improved to further enhance patient outcomes, and warrant further study of combination regimens and the real-world impact of TKI/IO combination therapy.

\section{ACKNOWLEDGEMENTS}

Funding. Sponsorship for this study as well as the journal's Rapid Service and Open Access fees were funded by EMD Serono, Inc., an affiliate of Merck KGaA, Darmstadt, Germany, as part of an alliance between Merck KGaA and Pfizer.

Medical Writing and Editorial Assistance. Medical writing support for this 
manuscript was provided by Michael Kane of STATinMED Research, and was funded by the study sponsors. Editorial support was provided by Chris Haddlesey of STATinMED Research, and was funded by the study sponsors.

Authorship. All named authors meet the International Committee of Medical Journal Editors (ICMJE) criteria for authorship for this article, take responsibility for the integrity of the work as a whole, and have given their approval for this version to be published. All authors had full access to all of the data in this study and take complete responsibility for the integrity of the data and accuracy of the data analysis.

Prior Presentation. Data from this study has been previously presented in poster format at ISPOR 2019 Annual Conference and at the 18th International Kidney Cancer Symposium (2019).

Disclosures. Abhijeet Bhanegaonkar is an employee of EMD Serono, Inc., an affiliate of Merck KGaA, Darmstadt, Germany, one of the study sponsors. Hemant Phatak, Vijay Kasturi, and Ying Zheng were employees of EMD Serono, Inc., at the time the study was conducted and are now employees of Acceleron Pharma, FerGene, and Sanofi, respectively. Ruth Kim and Stan Krulewicz are employees of Pfizer, Inc., one of the study sponsors. Shivani Pandya was an employee of STATinMED Research, a paid consultant to the study sponsors, at the time the study was conducted.

Compliance with Ethics Guidelines. This retrospective database analysis did not involve the collection, use, or transmittal of individual identifiable data. As such, institutional review board approval to conduct this study was not required, as it is considered exempt according to 45CFR46.101(b)(4): Existing Data \& Specimens No Identifiers. Both the dataset itself and the security of the offices where the data are housed meet the requirements of the Health Insurance Portability and Accountability Act of 1996.
Data Availability. The dataset supporting the conclusions in this article is available from the US Veterans Health Administration, which we had full permissions to access and use for this study. However, restrictions apply to the availability of these data, which were used under license for the current study, and so are not publicly available.

Open Access. This article is licensed under a Creative Commons Attribution-NonCommercial 4.0 International License, which permits any non-commercial use, sharing, adaptation, distribution and reproduction in any medium or format, as long as you give appropriate credit to the original author(s) and the source, provide a link to the Creative Commons licence, and indicate if changes were made. The images or other third party material in this article are included in the article's Creative Commons licence, unless indicated otherwise in a credit line to the material. If material is not included in the article's Creative Commons licence and your intended use is not permitted by statutory regulation or exceeds the permitted use, you will need to obtain permission directly from the copyright holder. To view a copy of this licence, visit http://creativecommons.org/licenses/bync/4.0/.

\section{REFERENCES}

1. Cancer Facts \& Figures 2019. American Cancer Society website. 2019. https://www.cancer.org/ content/dam/cancer-org/research/cancer-facts-andstatistics/annual-cancer-facts-and-figures/2019/ cancer-facts-and-figures-2019.pdf. Accessed 25 Nov 2019.

2. Gad MM, Saad AM, Al-Huseini MJ, Ruhban IA, Sonbol MB. Incidence and mortality of renal cell carcinoma in the US: a SEER-based study investigating trends over the last four decades. J Clin Oncol. 2018;36(6 Suppl):604.

3. Pal SK, Vanderwalde A, Hurria A, Figlin RA. Systemic therapies for metastatic renal cell carcinoma in older adults. Drugs Aging. 2011;28(8):635-49.

4. Gupta K, Miller JD, Li JZ, Russell MW, Charbonneau C. Epidemiologic and socioeconomic burden of 
metastatic renal cell carcinoma (mRCC): a literature review. Cancer Treat Rev. 2008;34(3):193-205.

5. Capitanio U, Bensalah K, Bex A, et al. Epidemiology of renal cell carcinoma. Eur Urol. 2019;75(1):74-84.

6. Saad AM, Gad MM, Al-Husseini MJ, Ruhban IA, Sonbol MB, Ho TH. Trends in renal-cell carcinoma incidence and mortality in the United States in the last 2 decades: a SEER-based study. Clin Genitourin Cancer. 2019;17(1):46-57.e5.

7. Cancer Stat Facts: Kidney and Renal Pelvis Cancer. National Institutes of Health, National Cancer Institute website. 2020. https://seer.cancer.gov/ statfacts/html/kidrp.html. Accessed 15 Apr 2020.

8. Borker R. Costs associated with adverse events in patients with metastatic renal cell carcinoma. J Med Econ. 2014;17(11):792-7.

9. Athar U, Gentile TC. Treatment options for metastatic renal cell carcinoma: a review. Can J Urol. 2008;15(2):3954-66.

10. Gong J, Miaa MC, Dizman N, Govindarajan A, Pal SK. Metastasis in renal cell carcinoma: biology and implications for therapy. Asian J Urol. 2016;3(4): 286-92.

11. Hutson TE, Bukowski RM, Rini BI, et al. Efficacy and safety of sunitinib in elderly patients with metastatic renal cell carcinoma. $\mathrm{Br} \mathrm{J}$ Cancer. 2014;110(5):1125-32.

12. Schey C, Meier G, Pan J. Metastatic renal cell cancer: an analysis of reimbursement decisions. Adv Ther. 2019;36(6):1266-78.

13. Escudier B, Eisen T, Stadler WM, et al. Sorafenib in advanced clear-cell renal-cell carcinoma. N Engl J Med. 2007;356:125-34.

14. Gill DM, Agarwal N, Vaishampayan U. Evolving treatment paradigm in metastatic renal cell carcinoma. Am Soc Oncol Educ Book. 2017;37:319-29.

15. Zahavi DJ, Weiner LM. Targeting multiple receptors to increase checkpoint blockade efficacy. Int J Mol Sci. $2019 ; 20(1): 158$.

16. Kammerer-Jacquet SF, Deleuze A, Saout J, et al. Targeting the PD-1/PD-L1 pathway in renal cell carcinoma. Int J Mol Sci. 2019;20(7):1692. https:// doi.org/10.3390/ijms20071692.

17. 2020 NCCN Guidelines: Kidney Cancer. National Comprehensive Cancer Care Network website. 2020. https://www.nccn.org/professionals/physician_ gls/pdf/kidney.pdf. Accessed 23 Sep 2020.
18. Hess G, Borker R, Fonseca E. Treatment patterns: targeted therapies indicated for first-line management of metastatic renal cell carcinoma in a realworld setting. Clin Genitour Cancer. 2013;11(2): 161-7.

19. Rini BI, Battle D, Figlin RA, et al. The Society for Immunotherapy of Cancer consensus statement on immunotherapy for the treatment of advanced renal cell carcinoma (RCC). J Immunother Cancer. 2019;7(1):354.

20. Lang K, Danchenko N, Gondek K, Schwartz B, Thompson D. The burden of illness associated with renal cell carcinoma in the United States. Urol Oncol. 2007;25(5):368-75.

21. Geynisman DM, Hu JC, Liu L, Shih YCT. Treatment patterns and costs for metastatic renal cell carcinoma patients with private insurance in the United States. Clin Genitour Cancer. 2015;13(2):e93-100.

22. United States Department of Veterans Affairs. Statistical Brief. Profile of Veterans. 2017. https://www. va.gov/vetdata/docs/SpecialReports/Profile_of_ Veterans_2017.pdf.

23. Nelson KM. The burden of obesity among a national probability sample of veterans. J Gen Intern Med. 2006;21(9):915-9.

24. Odani S, Agaku IT, Graffunder CM, Tynan MA, Armour BS. Tobacco product use among military veterans-United States, 2010-2015. MMWR Morb Mortal Wkly Rep. 2018;67(1):7-12.

25. Gillespie CD, Hurvitz KA. Prevalence of hypertension and controlled hypertension-United States, 2007-2010. MMWR Morb Mortal Wkly Rep. 2012;62(3):144-8.

26. About VHA. U.S. Department of Veterans Affairs website. 2019. https://www.va.gov/health/ aboutvha.asp. Accessed 23 Sep 2020.

27. Baser O, Xie L, Pesa J, Durkin M. Healthcare utilization and costs of veteran health administration patients with schizophrenia treated with paliperidone palmitate long-acting injection or oral atypical antipsychotics. J Med Econ. 2015;18(5):357-65.

28. Quan H, Sundarajan V, Halfon P, et al. Coding algorithms for defining comorbidities in ICD-9CDM and ICD-10 administrative data. Med Care. 2005;43(11):1130-9.

29. Klabunde CN, Potosky AL, Legler JM, Warren JL. Development of a comorbidity index using physician claims data. J Clin Epidemiol. 2000;53(12):1258-67. 
30. Brown DW. Smoking prevalence among US veterans. J Gen Intern Med. 2010;25(2):147-9.

31. Motzer RJ, Tannir NM, McDermott DF, et al. Nivolumab plus ipilimumab versus sunitinib in advanced renal-cell carcinoma. $\mathrm{N}$ Engl J Med. 2018;378(14):1277-90.

32. Motzer RJ, Penkov K, Haanen J, et al. Avelumab plus axitinib versus sunitinib for advanced renal-cell carcinoma. N Engl J Med. 2019;380(12):1103-15.

33. Choueiri TK, Motzer RJ, Rini BI, et al. Updated efficacy results from the JAVELIN Renal 101 trial: first-line avelumab plus axitinib versus sunitinib in patients with advanced renal cell carcinoma. Ann Oncol. 2020;31(8):1030-9.

34. Rini BI, Plimack ER, Stus V, et al. Pembrolizumab plus axitinib versus sunitinib for advanced renalcell carcinoma. N Engl J Med. 2019;380(12): 1116-27.

35. Shih YCT, Chien CR, Xu Y, Pan IW, Smith GL, Buchholz TA. Economic burden of renal cell carcinoma in the US: part II-an updated analysis. Pharmacoeconomics. 2011;29(4):331-41.
36. Chien CR, Geynisman DM, Kim B, Xu Y, Shih YT. Economic burden of renal cell carcinoma-part I: an updated review. Pharmacoeconomics. 2019;37(3): 301-31.

37. Bersanelli M, Leonardi F, Buti S. Spotlight on cabozantinib for previously untreated advanced renal cell carcinoma: evidence to date. Cancer Manag Res. 2018;10:3773-80.

38. Motzer RJ, Hutson TE, Cella D, et al. Pazopanib versus sunitinib in metastatic renal-cell carcinoma. N Engl J Med. 2013;369(8):722-31.

39. Hutson TE. Targeted therapies for the treatment of metastatic renal cell carcinoma: clinical evidence. Oncologist. 2011;16(Suppl 2):14-22.

40. Tannir NM, McDermott DF, Escudier B, et al. Overall survival and independent review of response in CheckMate 214 with 42-month followup: first-line nivolumab + ipilimumab versus sunitinib in patients with advanced renal cell carcinoma (aRCC). J Clin Oncol. 2020;38(6):609. 